PREPARED FOR THE U.S. DEPARTMENT OF ENERGY, UNDER CONTRACT DE-AC02-76CH03073

PPPL-3625

PPPL-3625

UC-70

Implications of the Electrostatic Approximation in the Beam Frame on the Nonlinear Vlasov-Maxwell Equations for Intense Beam Propagation

by

Ronald C. Davidson, W. Wei-li Lee, Hong Qin, and Edward Startsev

November 2001

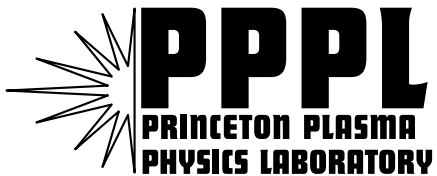

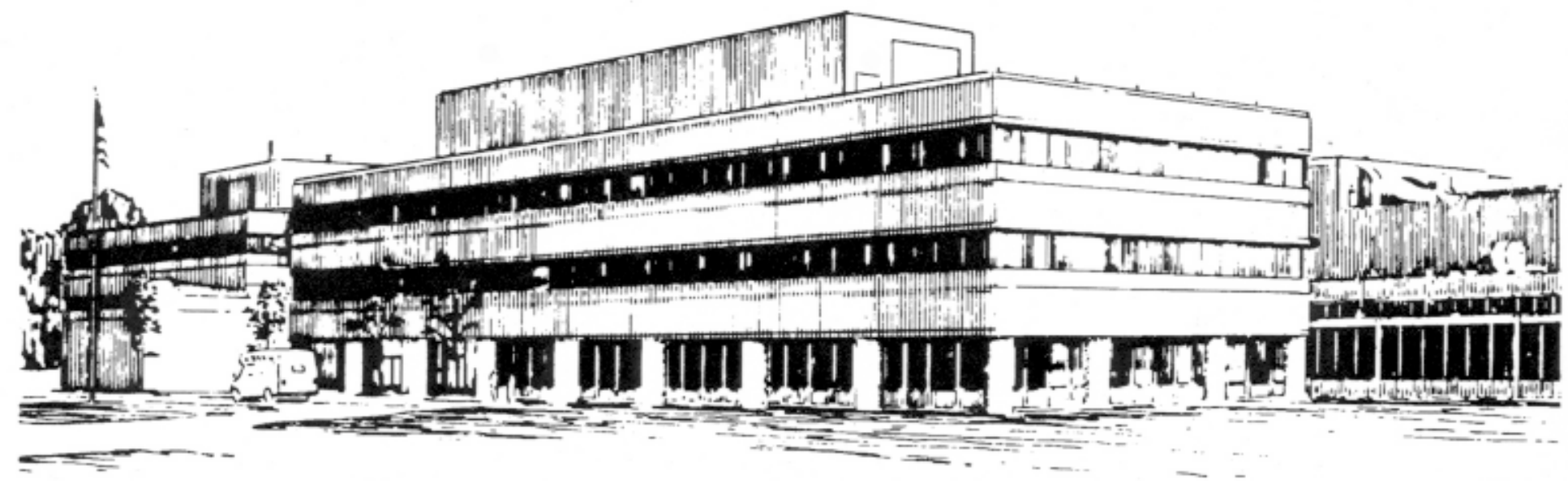

PRINCETON PLASMA PHYSICS LABORATORY PRINCETON UNIVERSITY, PRINCETON, NEW JERSEY 


\section{PPPL Reports Disclaimer}

This report was prepared as an account of work sponsored by an agency of the United States Government. Neither the United States Government nor any agency thereof, nor any of their employees, makes any warranty, express or implied, or assumes any legal liability or responsibility for the accuracy, completeness, or usefulness of any information, apparatus, product, or process disclosed, or represents that its use would not infringe privately owned rights. Reference herein to any specific commercial product, process, or service by trade name, trademark, manufacturer, or otherwise, does not necessarily constitute or imply its endorsement, recommendation, or favoring by the United States Government or any agency thereof. The views and opinions of authors expressed herein do not necessarily state or reflect those of the United States Government or any agency thereof.

\section{Availability}

This report is posted on the U.S. Department of Energy's Princeton Plasma Physics Laboratory Publications and Reports web site in Fiscal Year 2002. The home page for PPPL Reports and Publications is: http://www.pppl.gov/pub_report/

DOE and DOE Contractors can obtain copies of this report from:

U.S. Department of Energy

Office of Scientific and Technical Information

DOE Technical Information Services (DTIS)

P.O. Box 62

Oak Ridge, TN 37831

Telephone: (865) 576-8401

Fax: (865) 576-5728

Email: reports@adonis.osti.gov

This report is available to the general public from:

National Technical Information Service

U.S. Department of Commerce

5285 Port Royal Road

Springfield, VA 22161

Telephone: 1-800-553-6847 or

(703) 605-6000

Fax: (703) 321-8547

Internet: http://www.ntis.gov/ordering.htm 


\title{
Implications of the Electrostatic Approximation in the Beam Frame on the Nonlinear Vlasov-Maxwell Equatons for Intense Beam Propagation
}

\author{
Ronald C. Davidson, W. Wei-li Lee, Hong Qin and Edward Startsev \\ Plasma Physics Laboratory, Princeton University, Princeton, NJ 08543
}

\begin{abstract}
This paper develops a clear procedure for solving the nonlinear VlasovMaxwell equations for a one-component intense charged particle beam or finite-length charge bunch propagating through a cylindrical conducting pipe (radius $r=r_{w}=$ const.), and confined by an applied focusing force $\mathbf{F}_{f o c}$. In particular, the nonlinear Vlasov-Maxwell equations are Lorentz-transformed to the beam frame ('primed' variables) moving with axial velocity $V_{b}=\beta_{b} c=$ const. relative to the laboratory. In the beam frame, the particle motions are nonrelativistic for the applications of practical interest, already a major simplification. Then, in the beam frame, we make the electrostatic approximation $\left(\mathbf{E}_{s}^{\prime}=-\nabla^{\prime} \phi^{\prime}, \mathbf{E}_{T}^{\prime} \simeq 0 \simeq \mathbf{B}_{s}^{\prime}\right)$ which fully incorporates beam spacecharge effects, but neglects any fast electromagnetic processes with transverse polarization (e.g., light waves). The resulting Vlasov-Maxwell equations are then Lorentz-transformed back to the laboratory frame, and properties of the self-generated fields and resulting nonlinear Vlasov-Maxwell equations in the laboratory frame are discussed.
\end{abstract}

Typeset using REVTEX 


\section{INTRODUCTION}

Periodic focusing accelerators and transport systems [1-7] have a wide range of applications ranging from basic scientific research in high energy and nuclear physics, to applications such as coherent radiation sources, heavy ion fusion, tritium production, nuclear waste transmutation, and spallation neutron sources for materials and biological research [8,9]. At the high beam currents and charge densities of practical interest, of particular importance are the effects of the intense self fields produced by the beam space charge and current on determining the detailed equilibrium, stability and transport properties, and the nonlinear dynamics of the system. Through analytical studies based on the nonlinear Vlasov-Maxwell equations for the distribution function $f_{b}(\mathbf{x}, \mathbf{p}, t)$ and the self-generated electric and fields $\mathbf{E}_{s}(\mathbf{x}, t)$ and $\mathbf{B}_{s}(\mathbf{x}, t)$, and numerical simulations using particle-in-cell models and nonlinear perturbative simulation techniques, considerable progress has been made in developing an improved understanding of the collective processes and nonlinear beam dynamics characteristic of high-intensity beam propagation in periodic focusing and uniform focusing transport systems [10-28]. In almost all applications of the Vlasov-Maxwell equations to intense beam propagation, the analysis is carried out in the laboratory frame, and various simplifying approximations are made, ranging from the electrostatic-magnetostatic approximation [29], to the Darwin-model approximation [30-35] which neglects fast transverse electromagnetic perturbations.

Given the general importance of model assumptions in affecting the detailed outcome of calculations, in this paper we develop a clear procedure for solving the nonlinear VlasovMaxwell equations for a one-component intense charged particle beam or finite-length charge bunch propagating through a cylindrical conducting pipe (radius $r=r_{w}=$ const.), and confined by an applied focusing force $\mathbf{F}_{f o c}$. In particular, the nonlinear Vlasov-Maxwell equations are Lorentz-transformed to the beam frame ('primed' variables) moving with axial velocity $V_{b}=\beta_{b} c=$ const. relative to the laboratory [24]. In the beam frame, the particle motions are nonrelativistic for the applications of practical interest, already a major simpli- 
fication. Then, in the beam frame, we make the electrostatic approximation $\left(\mathbf{E}_{s}^{\prime}=-\nabla^{\prime} \phi^{\prime}\right.$, $\mathbf{E}_{T}^{\prime} \simeq 0 \simeq \mathbf{B}_{s}^{\prime}$ ) which fully incorporates beam space-charge effects, but neglects any fast electromagnetic processes with transverse polarization (e.g., light waves). The resulting Vlasov-Maxwell equations are then Lorentz-transformed back to the laboratory frame, and properties of the self-generated fields and resulting nonlinear Vlasov-Maxwell equations in the laboratory frame are discussed.

\section{VLASOV-MAXWELL EQUATIONS AND TRANSFORMATION TO THE BEAM FRAME}

In the present analysis, we consider an intense charged particle beam with characteristic transverse dimensions $a$ and $b$ propagating in the $z$-direction with average axial velocity $V_{b}=\beta_{b} c=$ const. and characteristic directed kinetic energy $\left(\gamma_{b}-1\right) m_{b} c^{2}$. Here, $c$ is the speed of light in vacuo, $\gamma_{b}=\left(1-\beta_{b}^{2}\right)^{-1 / 2}$ is the relativistic mass factor, and $e_{b}$ and $m_{b}$ are the charge and rest mass, respectively, of a beam particle. A perfectly conducting cylindrical wall is located at radius $r=r_{w}$, where $r=\left(x^{2}+y^{2}\right)^{1 / 2}$ is the radial distance from the beam axis. The particle motion in the beam frame ('primed' coordinates) is assumed to be nonrelativistic with $\left|\mathbf{v}^{\prime}\right| \ll c$. Furthermore, the beam current density and charge density are allowed to be arbitrarily large, subject only to the requirement that the beam particles be confined by the applied focusing fields $\mathbf{E}_{f o c}(\mathbf{x}, t)$ and $\mathbf{B}_{f o c}(\mathbf{x}, t)$. The specific forms of $\mathbf{E}_{f o c}(\mathbf{x}, t)$ and $\mathbf{B}_{f o c}(\mathbf{x}, t)$ of course depend on the particular focusing field configuration under consideration (quadrupole, solenoidal, rf, etc.). Finally, in the present analysis, the beam can be continuous in the $z$-direction, or correspond to a finite-length charge bunch.

Within the context of the above assumptions, a complete description of the collective processes and nonlinear dynamics of the charged particle beam is provided by the nonlinear Vlasov-Maxwell equations [1], which describe the evolution of the distribution function $f_{b}(\mathbf{x}, \mathbf{p}, t)$ in the six-dimensional laboratory-frame phase space $(\mathbf{x}, \mathbf{p})$, and the corresponding self fields, $\mathbf{E}_{s}(\mathbf{x}, t)$ and $\mathbf{B}_{s}(\mathbf{x}, t)$, generated self-consistently by the beam space charge and 
current. In laboratory-frame variables, the nonlinear Vlasov-Maxwell equations describing the self-consistent evolution of $f_{b}(\mathbf{x}, \mathbf{p}, t), \mathbf{E}_{s}(\mathbf{x}, t)$ and $\mathbf{B}_{s}(\mathbf{x}, t)$ can be expressed as

$$
\frac{\partial f_{b}}{\partial t}+\mathbf{v} \cdot \frac{\partial f_{b}}{\partial \mathbf{x}}+\left[\mathbf{F}_{f o c}+e_{b}\left(\mathbf{E}_{s}+\frac{1}{c} \mathbf{v} \times \mathbf{B}_{s}\right)\right] \cdot \frac{\partial f_{b}}{\partial \mathbf{p}}=0
$$

and

$$
\begin{gathered}
\nabla \cdot \mathbf{E}_{s}=4 \pi e_{b} \int d^{3} p f_{b} \\
\nabla \times \mathbf{B}_{s}=\frac{1}{c} 4 \pi e_{b} \int d^{3} p \mathbf{v} f_{b}+\frac{1}{c} \frac{\partial \mathbf{E}_{s}}{\partial t} \\
\nabla \times \mathbf{E}_{s}=-\frac{1}{c} \frac{\partial \mathbf{B}_{s}}{\partial t} \\
\nabla \cdot \mathbf{B}_{s}=0
\end{gathered}
$$

Here, $\mathbf{F}_{f o c}=e_{b}\left(\mathbf{E}_{f o c}+c^{-1} \mathbf{v} \times \mathbf{B}_{f o c}\right)$ is the applied focusing force in the laboratory frame, and the velocity $\mathbf{v}$ and momentum $\mathbf{p}$ are related by $\mathbf{p}=\gamma m_{b} \mathbf{v}$, where $\gamma=\left(1+\mathbf{p}^{2} / m_{b}^{2} c^{2}\right)^{1 / 2}$. The Vlasov equation (1) describes the incompressible evolution of the distribution function $f_{b}(\mathbf{x}, \mathbf{p}, t)$ in the six-dimensional phase space $(\mathbf{x}, \mathbf{p})$ as the beam particles interact with the applied focusing fields, $\mathbf{E}_{f o c}(\mathbf{x}, t)$ and $\mathbf{B}_{f o c}(\mathbf{x}, t)$, and the average self fields, $\mathbf{E}_{s}(\mathbf{x}, t)$ and $\mathbf{B}_{s}(\mathbf{x}, t)$, generated by the beam particles. Note that the Vlasov equation (1) is highly nonlinear because $\mathbf{E}_{s}(\mathbf{x}, t)$ and $\mathbf{B}_{s}(\mathbf{x}, t)$ are determined self-consistently in terms of the beam charge density, $\rho_{b}(\mathbf{x}, t)=e_{b} \int d^{3} p f_{b}(\mathbf{x}, \mathbf{p}, t)$, and current density, $\mathbf{J}_{b}(\mathbf{x}, t)=e_{b} \int d^{3} p \mathbf{v} f_{b}(\mathbf{x}, \mathbf{p}, t)$, from Maxwell's equations (2)-(5). Here, $n_{b}(\mathbf{x}, t)=\int d^{3} p f_{b}(\mathbf{x}, \mathbf{p}, t)$ is the number density of beam particles.

The Vlasov-Maxwell equations (1)-(5) can of course be analyzed directly in laboratoryframe variables. However, for a beam consisting of a single charge component (the case considered here), there is considerable advantage to transforming to the beam frame moving with axial velocity $V_{b}=\beta_{b} c$ relative to the laboratory. In the beam frame the particle motion is nonrelativistic $\left(\left|\mathbf{v}^{\prime}\right| \ll c\right)$ by assumption, which results in a welcome simplification of the 
corresponding Vlasov-Maxwell equations in the beam frame. The Lorentz transformation $[36,37]$ relating the primed variables $\left(\mathbf{x}^{\prime}, \mathbf{p}^{\prime}, t^{\prime}\right)$ in the beam frame to the unprimed variables $(\mathbf{x}, \mathbf{p}, t)$ in the laboratory frame is given by

$$
\begin{aligned}
& x^{\prime}=x, \quad y^{\prime}=y, \quad z^{\prime}=\gamma_{b}\left(z-V_{b} t\right), \\
& t^{\prime}=\gamma_{b}\left(t-V_{b} z / c^{2}\right), \\
& p_{x}^{\prime}=p_{x}, \quad p_{y}^{\prime}=p_{y}, \quad p_{z}^{\prime}=\gamma_{b}\left(p_{z}-\gamma m_{b} V_{b}\right), \\
& \gamma^{\prime}=\gamma_{b}\left(\gamma-V_{b} p_{z} / m_{b} c^{2}\right),
\end{aligned}
$$

where $\gamma_{b}=\left(1-V_{b}^{2} / c^{2}\right)^{-1 / 2}$. Here, the particle momentum and velocity are related by $\mathbf{p}=\gamma m_{b} \mathbf{v}$ and $\mathbf{p}^{\prime}=\gamma^{\prime} m_{b} \mathbf{v}^{\prime}$, where $\gamma=\left(1+\mathbf{p}^{2} / m_{b}^{2} c^{2}\right)^{1 / 2}$ and $\gamma^{\prime}=\left(1+\mathbf{p}^{\prime 2} / m_{b}^{2} c^{2}\right)^{1 / 2}$ are the kinematic mass factors. In the beam frame, the nonlinear Vlasov equation for the distribution function $f_{b}^{\prime}\left(\mathbf{x}^{\prime}, \mathbf{p}^{\prime}, t^{\prime}\right)$ can be expressed as [24]

$$
\frac{\partial f_{b}^{\prime}}{\partial t^{\prime}}+\mathbf{v}^{\prime} \cdot \frac{\partial f_{b}^{\prime}}{\partial \mathbf{x}^{\prime}}+\left[\mathbf{F}_{f o c}^{\prime}+e_{b}\left(\mathbf{E}_{s}^{\prime}+\frac{1}{c} \mathbf{v}^{\prime} \times \mathbf{B}_{s}^{\prime}\right)\right] \cdot \frac{\partial f_{b}^{\prime}}{\partial \mathbf{p}^{\prime}}=0
$$

In Eq. (7), $\mathbf{E}_{s}^{\prime}\left(\mathbf{x}^{\prime}, t\right)$ and $\mathbf{B}_{s}^{\prime}\left(\mathbf{x}^{\prime}, t^{\prime}\right)$ are the self-generated fields in the beam frame, and we approximate $\gamma^{\prime}=1+\mathbf{p}^{\prime 2} / 2 m_{b}^{2} c^{2}$ and $\mathbf{p}^{\prime}=m_{b} \mathbf{v}^{\prime}$ because the particle motion in the beam frame is assumed to be nonrelativistic. Furthermore, $\mathbf{F}_{f o c}^{\prime}=e_{b}\left(\mathbf{E}_{f o c}^{\prime}+c^{-1} \mathbf{v}^{\prime} \times \mathbf{B}_{f o c}^{\prime}\right)$ is the applied focusing force on a particle in the beam frame. Here, the applied electric and magnetic fields transform according to $\mathbf{E}_{f o c}^{\prime}=\left[E_{z} \hat{\mathbf{e}}_{z}+\gamma_{b}\left(E_{x} \hat{\mathbf{e}}_{x}+E_{y} \hat{\mathbf{e}}_{y}\right)+\gamma_{b} c^{-1} V_{b} \hat{\mathbf{e}}_{z} \times \mathbf{B}\right]_{f o c}$ and $\mathbf{B}_{f o c}^{\prime}=\left[B_{z} \hat{\mathbf{e}}_{z}+\gamma_{b}\left(B_{x} \hat{\mathbf{e}}_{x}+B_{y} \hat{\mathbf{e}}_{y}\right)-\gamma_{b} c^{-1} V_{b} \hat{\mathbf{e}}_{z} \times \mathbf{E}\right]_{f o c}$, which determines $\mathbf{E}_{f o c}^{\prime}$ and $\mathbf{B}_{f o c}^{\prime}$ directly in terms of the applied focusing fields in the laboratory frame and the Lorentz transformation in Eq. (6).

Maxwell's equations in the beam frame of course are similar in form to Eqs. (2)-(5), and relate the self-generated electric and magnetic fields, $\mathbf{E}_{s}^{\prime}\left(\mathbf{x}^{\prime}, t^{\prime}\right)$ and $\mathbf{B}_{s}^{\prime}\left(\mathbf{x}^{\prime}, t^{\prime}\right)$, to the distribution function $f_{b}^{\prime}\left(\mathbf{x}^{\prime}, \mathbf{p}, t^{\prime}\right)$. For present purposes, it is convenient to introduce the scalar and vector potentials, $\phi^{\prime}\left(\mathbf{x}^{\prime}, t^{\prime}\right)$ and $\mathbf{A}^{\prime}\left(\mathbf{x}^{\prime}, t^{\prime}\right)$, and express

$$
\mathbf{E}_{s}^{\prime}=\mathbf{E}_{L}^{\prime}+\mathbf{E}_{T}^{\prime}=-\frac{\partial}{\partial \mathbf{x}^{\prime}} \phi^{\prime}-\frac{1}{c} \frac{\partial}{\partial t^{\prime}} \mathbf{A}^{\prime},
$$




$$
\mathbf{B}_{s}^{\prime}=\frac{\partial}{\partial \mathbf{x}^{\prime}} \times \mathbf{A}^{\prime}
$$

where $\mathbf{E}_{L}^{\prime}=-\nabla^{\prime} \phi^{\prime}$ is the longitudinal electric field, $\mathbf{E}_{T}^{\prime}=c^{-1} \partial \mathbf{A}^{\prime} / \partial t^{\prime}$ is the transverse electric field, and the Coulomb gauge condition with $\nabla^{\prime} \cdot \mathbf{A}^{\prime}=0$ is assumed. From Eq. (8), the Maxwell equations $\nabla^{\prime} \cdot \mathbf{B}_{s}^{\prime}=0$ and $\nabla^{\prime} \times \mathbf{E}_{s}^{\prime}=-c^{-1} \partial \mathbf{B}_{s}^{\prime} / \partial t^{\prime}$ are automatically satisfied, and Poisson's equation and the $\nabla^{\prime} \times \mathbf{B}_{s}^{\prime}$ Maxwell equation in the beam frame are readily expressed as [24]

$$
\begin{gathered}
\nabla^{\prime 2} \phi^{\prime}=-4 \pi e_{b} \int d^{3} p^{\prime} f_{b}^{\prime} \\
\nabla^{\prime 2} \mathbf{A}^{\prime}=-\frac{1}{c} 4 \pi e_{b} \int d^{3} p^{\prime} \mathbf{v}^{\prime} f_{b}^{\prime}+\frac{1}{c^{2}} \frac{\partial^{2} \mathbf{A}^{\prime}}{\partial t^{\prime 2}}+\frac{1}{c} \nabla^{\prime} \frac{\partial \phi^{\prime}}{\partial t^{\prime}}
\end{gathered}
$$

where use has been made of $\nabla^{\prime} \cdot \mathbf{A}^{\prime}=0$. In Eqs. (9) and (10), note that the electrostatic potential $\phi^{\prime}\left(\mathbf{x}^{\prime}, t^{\prime}\right)$ is determined self-consistently in terms of the beam charge density $\rho_{b}^{\prime}\left(\mathbf{x}^{\prime}, t^{\prime}\right)=e_{b} n_{b}^{\prime}\left(\mathbf{x}^{\prime}, t^{\prime}\right)=e_{b} \int d^{3} p^{\prime} f_{b}^{\prime}\left(\mathbf{x}^{\prime}, \mathbf{p}^{\prime}, t^{\prime}\right)$ from Eq. (9), and $\mathbf{A}^{\prime}\left(\mathbf{x}^{\prime}, t^{\prime}\right)$ is determined in terms of the beam current density $\mathbf{J}_{b}^{\prime}\left(\mathbf{x}^{\prime}, t^{\prime}\right)=e_{b} n_{b}^{\prime}\left(\mathbf{x}^{\prime}, t^{\prime}\right) \mathbf{V}_{b}^{\prime}\left(\mathbf{x}^{\prime}, t^{\prime}\right)=e_{b} \int d^{3} p^{\prime} \mathbf{v}^{\prime} f_{b}^{\prime}\left(\mathbf{x}^{\prime}, \mathbf{p}^{\prime}, t^{\prime}\right)$ from Eq. (10). Here, $n_{b}^{\prime}\left(\mathbf{x}^{\prime}, t^{\prime}\right)$ is the local number density and $\mathbf{V}_{b}^{\prime}\left(\mathbf{x}^{\prime}, t^{\prime}\right)$ is the local average flow velocity of particles in the beam frame, and $\mathbf{v}^{\prime}=\mathbf{p}^{\prime} / m_{b}$ is the (nonrelativistic) particle velocity. Note that Poisson's equation (9) can be viewed as an initial condition to Eq. (10), which if true at $t^{\prime}=0$ remains true at all subsequent $t^{\prime}$. This follows upon taking the divergence of Eq. (10) and making use of the Coulomb gauge condition $\nabla^{\prime} \cdot \mathbf{A}^{\prime}=0$. This readily gives

$$
\frac{1}{c} \frac{\partial}{\partial t^{\prime}} \nabla^{\prime 2} \phi^{\prime}-\frac{1}{c} 4 \pi e_{b} \nabla^{\prime} \cdot\left(n_{b}^{\prime} \mathbf{V}_{b}^{\prime}\right)=\frac{1}{c} \frac{\partial}{\partial t^{\prime}}\left[\nabla^{\prime 2} \phi^{\prime}+4 \pi e_{b} n_{b}^{\prime}\right]=0
$$

where use has been made of $\partial n_{b}^{\prime} / \partial t^{\prime}+\nabla^{\prime} \cdot\left(n_{b}^{\prime} \mathbf{V}_{b}^{\prime}\right)=0$. It follows trivially from Eq. (11) that if Poisson's equation (9) is satisfied initially at $t^{\prime}=0$, then it remains true at all subsequent times $t^{\prime}$.

For the boundary conditions at the perfectly conducting cylindrical wall at radius $r=r^{\prime}=r_{w}$, we impose the requirements that the tangential electric field and the normal 
magnetic field vanish. That is, $\left[E_{z}\right]_{r=r_{w}}=\left[E_{\theta}\right]_{r=r_{w}}=\left[B_{r}\right]_{r=r_{w}}=0$, where $E_{z}, E_{\theta}$ and $B_{r}$ denote field components in cylindrical polar coordinates $(r, \theta, z)$ in the laboratory frame. In the beam frame, the corresponding field components are given by $E_{z}^{\prime}=E_{z}, B_{r}^{\prime}=\gamma_{b}\left(B_{r}+V_{b} E_{\theta} / c\right)$, and $E_{\theta}^{\prime}=\gamma_{b}\left(E_{\theta}+V_{b} B_{r} / c\right)$. Therefore, the corresponding boundary conditions at the conducting wall $r^{\prime}=r_{w}$ in the beam frame are also given by $\left[E_{z}^{\prime}\right]_{r^{\prime}=r_{w}}=\left[E_{\theta}^{\prime}\right]_{r^{\prime}=r_{w}}=\left[B_{r}^{\prime}\right]_{r^{\prime}=r_{w}}=0$. Expressed in terms of the scalar and vector potentials, $\phi^{\prime}\left(\mathbf{x}^{\prime}, t^{\prime}\right)$ and $\mathbf{A}^{\prime}\left(\mathbf{x}^{\prime}, t^{\prime}\right)$, these boundary conditions can be expressed as

$$
\phi^{\prime}\left(r^{\prime}=r_{w}, \theta^{\prime}, z^{\prime}, t^{\prime}\right)=A_{z}^{\prime}\left(r^{\prime}=r_{w}, \theta^{\prime}, z^{\prime}, t^{\prime}\right)=A_{\theta}^{\prime}\left(r^{\prime}=r_{w}, \theta^{\prime}, z^{\prime}, t^{\prime}\right)=\text { const. }
$$

where $\left(r^{\prime}, \theta^{\prime}, z^{\prime}\right)$ are cylindrical polar coordinates in the beam frame, with $x^{\prime}=r^{\prime} \cos \theta^{\prime}$ and $y^{\prime}=r^{\prime} \sin \theta^{\prime}$.

The nonlinear Vlasov-Maxwell equations (7), (9) and (10) in the beam frame, subject to the boundary conditions in Eq. (12), are fully equivalent to the Vlasov-Maxwell equations (1)-(5) in the laboratory frame, and provide a complete description of the collective processes and nonlinear dynamics of intense beam propagation. Equations (7), (9) and (10) can be used to investigate detailed equilibrium and stability properties in the beam frame for a wide range of system parameters and choices of applied field configurations. Moreover, as noted earlier, because the particle motion in the beam frame is nonrelativistic, a detailed investigation of Eqs. (7), (9) and (10) is more tractable analytically and numerically than the corresponding Vlasov-Maxwell equations (1)-(5) in the laboratory frame. Furthermore, once the solutions for $f_{b}^{\prime}\left(\mathbf{x}^{\prime}, \mathbf{p}^{\prime}, t^{\prime}\right), \phi^{\prime}\left(\mathbf{x}^{\prime}, t^{\prime}\right)$ and $\mathbf{A}^{\prime}\left(\mathbf{x}^{\prime}, t^{\prime}\right)$ are obtained in the beam frame, the corresponding solutions are readily obtained in the laboratory frame. For example, the variables $\left(\mathbf{x}^{\prime}, \mathbf{p}^{\prime}, t^{\prime}\right)$ are related to $(\mathbf{x}, \mathbf{p}, t)$ by the Lorentz transformation in Eq. (6). Furthermore, the scalar and vector potentials $\phi(\mathbf{x}, t)$ and $\mathbf{A}(\mathbf{x}, t)$ in the laboratory frame are related to the potentials $\phi^{\prime}\left(\mathbf{x}^{\prime}, t^{\prime}\right)$ and $\mathbf{A}^{\prime}\left(\mathbf{x}^{\prime}, t^{\prime}\right)$ in the beam frame by the transformation $[24,36]$

$$
\begin{gathered}
\phi=\gamma_{b}\left(\phi^{\prime}+V_{b} A_{z}^{\prime} / c\right), \\
A_{x}=A_{x}^{\prime}, A_{y}=A_{y}^{\prime},
\end{gathered}
$$




$$
A_{z}=\gamma_{b}\left(A_{z}^{\prime}+V_{b} \phi^{\prime} / c\right)
$$

where the arguments $\left(\mathbf{x}^{\prime}, t^{\prime}\right)$ are related to $(\mathbf{x}, t)$ by Eq. (6).

\section{IMPLICATIONS OF ELECTROSTATIC APPROXIMATION IN THE BEAM FRAME}

The introduction of the Coulomb gauge condition $\left(\nabla^{\prime} \cdot \mathbf{A}^{\prime}=0\right)$ and the resulting forms of Maxwell's equations (9) and (10) also make ancillary approximations more transparent in the beam frame. In the following analysis, we consider such a case corresponding to the electrostatic approximation in the beam frame. In particular, it is assumed that the electromagnetic field components with transverse polarization, $\mathbf{E}_{T}^{\prime}=-c^{-1} \partial \mathbf{A}^{\prime} / \partial t^{\prime}$ and $\mathbf{B}_{s}^{\prime}=$ $\nabla^{\prime} \times \mathbf{A}^{\prime}$, are negligibly small in comparison with the longitudinal electric field, $\mathbf{E}_{L}^{\prime}=-\nabla^{\prime} \phi^{\prime}$. In this case, we approximate

$$
\begin{aligned}
\mathbf{A}^{\prime} & =0, \\
\mathbf{E}_{T}^{\prime} & =0=\mathbf{B}_{s}^{\prime},
\end{aligned}
$$

and the nonlinear Vlasov equation (7) in the beam frame becomes

$$
\frac{\partial f_{b}^{\prime}}{\partial t^{\prime}}+\mathbf{v}^{\prime} \cdot \frac{\partial f_{b}^{\prime}}{\partial \mathbf{x}^{\prime}}+\left[\mathbf{F}_{f o c}^{\prime}-e_{b} \nabla^{\prime} \phi^{\prime}\right] \cdot \frac{\partial f_{b}^{\prime}}{\partial \mathbf{p}^{\prime}}=0
$$

Of course the scalar potential $\phi^{\prime}\left(\mathbf{x}^{\prime}, t^{\prime}\right)$ occurring in Eq. (15) is determined self-consistently in terms of the charge density $e_{b} \int d^{3} p^{\prime} f_{b}^{\prime}\left(\mathbf{x}^{\prime}, \mathbf{p}^{\prime}, t^{\prime}\right)$ from Poison's equation (9). By virtue of Eq. (14), we have neglected any fast electromagnetic processes in the beam frame with transverse polarization (e.g., light waves), and it is assumed that the current carried by the particles in the beam frame is sufficiently small that the self-generated transverse field components $\mathbf{E}_{T}^{\prime}$ and $\mathbf{B}_{s}^{\prime}$ can be neglected. Equations (9) and (15) of course include the full influence of space-charge effects in the beam frame through the longitudinal electric field $\mathbf{E}_{L}^{\prime}=-\nabla^{\prime} \phi^{\prime}$. 
The nonlinear Vlasov-Poisson equations (9) and (15) constitute a closed description of the collective processes and nonlinear dynamics of the distribution function $f_{b}^{\prime}\left(\mathbf{x}^{\prime}, \mathbf{p}^{\prime}, t^{\prime}\right)$ and space-charge potential $\phi^{\prime}\left(\mathbf{x}^{\prime}, t^{\prime}\right)$ in the beam frame, valid in the electrostatic approximation. As such Eqs. (9) and (15) can be used to investigate detailed equilibrium and electrostatic stability properties for a wide range of system parameters and choices of focusing field configurations. The purpose of this paper is not to solve Eqs. (9) and (15) in detail. Rather, let us assume that the solutions to Eqs. (9) and (15) have been obtained in the beam frame (these could be analytical or numerical solutions), and pose the question: What are the properties of the corresponding solutions in the laboratory frame? The variables $\left(\mathbf{x}^{\prime}, \mathbf{p}^{\prime}, t^{\prime}\right)$ and $(\mathbf{x}, \mathbf{p}, t)$ of course transform according to Eq. (6). Furthermore, once $\phi^{\prime}\left(\mathbf{x}^{\prime}, t^{\prime}\right)$ is determined in the beam frame, field quantities in the laboratory frame are readily obtained by making use of Eqs. (13) and (14). Substituting $\mathbf{A}^{\prime}=0$ into Eq. (13) readily gives

$$
\begin{gathered}
\phi=\gamma_{b} \phi^{\prime}, \\
A_{x}=0=A_{y}, \\
A_{z}=\gamma_{b} \beta_{b} \phi^{\prime}=\beta_{b} \phi,
\end{gathered}
$$

where $\beta_{b}=V_{b} / c$. In Eq. (16), the arguments $\left(\mathbf{x}^{\prime}, t^{\prime}\right)$ and $(\mathbf{x}, t)$ are related by Eq. (6), so Eq. (16) gives directly

$$
\phi(x, y, z, t)=\gamma_{b} \phi^{\prime}\left[x, y, \gamma_{b}\left(z-V_{b} t\right), \gamma_{b}\left(t-V_{b} z / c^{2}\right)\right],
$$

as well as $A_{x}=A_{y}=0$, and

$$
A_{z}(x, y, z, t)=\beta_{b} \phi(x, y, z, t) .
$$

Note from Eqs. (16) and (17) that the $\left(x^{\prime}, y^{\prime}\right)$ and $(x, y)$ dependences of $\phi^{\prime}$ and $\phi$ are identical, whereas the $\left(z^{\prime}, t^{\prime}\right)$ and $(z, t)$ dependences transform according to Eq. (6). In terms of Fourier-Laplace variables $\left(k_{z}, \omega\right)$ and $\left(k_{z}^{\prime}, \omega^{\prime}\right)$, Eq. (17) leads directly to the familiar relations $[36,37]$ 


$$
\begin{gathered}
k_{z}=\gamma_{b}\left(k_{z}^{\prime}+\frac{V_{b}}{c^{2}} \omega^{\prime}\right), \\
\omega=\gamma_{b}\left(\omega^{\prime}+k_{z}^{\prime} V_{b}\right) .
\end{gathered}
$$

That is, if the potential $\phi^{\prime}\left(\mathbf{x}^{\prime}, t^{\prime}\right)$ has axial wavenumber $k_{z}^{\prime}$ and frequency $\omega^{\prime}$ in the beam frame, then the corresponding axial wavenumber $k_{z}$ and frequency $\omega$ in the laboratory frame are given by Eq. (19). Of course the inverse transformation to Eq. (19) is obtained by interchanging $\left(k_{z}, \omega\right)$ and $\left(k_{z}^{\prime}, \omega^{\prime}\right)$, and making the replacement $V_{b} \rightarrow-V_{b}$.

Equations (16)-(18) allow us to determine the self-generated fields, $\mathbf{E}_{s}=-\nabla \phi-$ $c^{-1} \partial \mathbf{A} / \partial t$ and $\mathbf{B}_{s}=\nabla \times \mathbf{A}$, in the laboratory frame, consistent with the electrostatic approximation, $\mathbf{E}_{s}^{\prime}=\mathbf{E}_{L}^{\prime}=-\nabla^{\prime} \phi^{\prime}$, in the beam frame. It follows directly that $\mathbf{B}_{s}=\nabla \times A_{z} \hat{\mathbf{e}}_{z}$ has components

$$
\begin{aligned}
B_{x} & =\frac{\partial A_{z}}{\partial y}=\beta_{b} \frac{\partial \phi}{\partial y}, \\
B_{y} & =-\frac{\partial A_{z}}{\partial x}=-\beta_{b} \frac{\partial \phi}{\partial x}, \\
B_{z} & =0 .
\end{aligned}
$$

Furthermore, the self-generated electric field $\mathbf{E}_{s}=-\nabla \phi-c^{-1}\left(\partial A_{z} / \partial t\right) \hat{\mathbf{e}}_{z}$ can be expressed as

$$
\begin{aligned}
& E_{x}=-\frac{\partial \phi}{\partial x}, \quad E_{y}=-\frac{\partial \phi}{\partial y}, \\
& E_{z}=-\frac{\partial \phi}{\partial z}-\frac{1}{c} \frac{\partial A_{z}}{\partial t}=-\frac{\partial \phi}{\partial z}-\frac{\beta_{b}}{c} \frac{\partial \phi}{\partial t} .
\end{aligned}
$$

As would be expected, even though there is no magnetic field in the beam frame $\left(\mathbf{B}_{s}^{\prime}=0\right)$ by assumption, in the laboratory frame the beam carries an axial current, and there is a transverse magnetic field generated with components, $B_{x}=\partial A_{z} / \partial y$ and $B_{y}=-\partial A_{z} / \partial x$, where $A_{z}=\beta_{b} \phi$ according to Eq. (18). Furthermore, in addition to the longitudinal electric field $\mathbf{E}_{L}=-\nabla \phi$, it follows from Eq. (21) that there is an inductive electric field $E_{T z}=$ $-c^{-1} \partial A_{z} / \partial t$ in the laboratory frame, where $A_{z}=\beta_{b} \phi$. As noted earlier, the nonlinear Vlasov-Poisson equations (9) and (15) can be solved in the beam frame, and then Eqs. (6), 
(16), (20) and (21) can be used to determine the corresponding distribution function and self-generated fields in the laboratory frame.

While Eqs. (9) and (15) provide a complete description of the system in the electrostatic aproximation in the beam frame, it is nonetheless interesting to examine the implications of the electrostatic approximation in the beam frame for the corresponding Vlasov-Maxwell equations in the laboratory frame. First, making use of Eqs. (20) and (21), it follows that the force on a beam particle in the laboratory frame due to the self-generated fields, $\mathbf{F}=e_{b}\left(\mathbf{E}_{s}+c^{-1} \mathbf{v} \times \mathbf{B}_{s}\right)$, can be expressed as $\mathbf{F}=\mathbf{F}_{\perp}+F_{z} \hat{\mathbf{e}}_{z}$, where

$$
\begin{aligned}
\mathbf{F}_{\perp} & =e_{b}\left(-\nabla_{\perp} \phi+\frac{1}{c}\left[\mathbf{v} \times\left(\nabla A_{z} \times \hat{e}_{z}\right)\right]_{\perp}\right) \\
& =-e_{b} \nabla_{\perp}\left(\phi-\frac{1}{c} v_{z} A_{z}\right)
\end{aligned}
$$

denotes the perpendicular force in the $x-y$ plane, and

$$
\begin{aligned}
F_{z} & =e_{b}\left(-\frac{\partial \phi}{\partial z}-\frac{1}{c} \frac{\partial A_{z}}{\partial t}-\frac{1}{c} \mathbf{v}_{\perp} \cdot \nabla_{\perp} A_{z}\right) \\
& =-e_{b}\left[\frac{\partial}{\partial z}\left(\phi-\frac{1}{c} v_{z} A_{z}\right)+\frac{1}{c}\left(\frac{\partial}{\partial t}+\mathbf{v} \cdot \nabla\right) A_{z}\right]
\end{aligned}
$$

is the axial force. Here, $\nabla_{\perp}=\hat{\mathbf{e}}_{x} \partial / \partial x+\hat{\mathbf{e}}_{y} \partial / \partial y$ is the perpendicular spatial gradient, and $A_{z}=\beta_{b} \phi$ follows from Eq. (18). Equations (22) and (23) can be substituted into the laboratory-frame Vlasov equation (1). One important simplification occurs in this regard. The characteristics of the Vlasov equation (1) are the single-particle orbits in the selfgenerated fields. For example, the coefficient of $\partial f_{b} / \partial p_{z}$ is $d p_{z} / d t=F_{z}$. From Eq. (23), introducing the axial canonical momentum $P_{z}=p_{z}+\left(e_{b} / c\right) A_{z}$, and making use of $(d / d t) A_{z}=$ $(\partial / \partial t+\mathbf{v} \cdot \nabla) A_{z}$, it follows that $d P_{z} / d t=-e_{b}(\partial / \partial z)\left(\phi-v_{z} A_{z} / c\right)$. Therefore, if we change variables from $\left(x, y, z, p_{x}, p_{y}, p_{z}, t\right)$ to $\left(x, y, z, P_{x}, P_{y}, P_{z}, t\right)$, where $P_{x}=p_{x}$ and $P_{y}=p_{y}$ (because $\left.A_{x}=0=A_{y}\right)$ and $P_{z}=p_{z}+\left(e_{b} / c\right) A_{z}$, it follows from Eqs. (1), (22) and (23) that the nonlinear Vlasov equation for the distribution function $f_{b}(\mathbf{x}, \mathbf{P}, t)$ in the laboratory frame can be expressed in the compact form

$$
\frac{\partial f_{b}}{\partial t}+\mathbf{v} \cdot \frac{\partial f_{b}}{\partial \mathbf{x}}+\left[\mathbf{F}_{f o c}-e_{b}\left(1-\frac{v_{z}}{c} \beta_{b}\right) \nabla \phi\right] \cdot \frac{\partial f_{b}}{\partial \mathbf{P}}=0
$$


In obtaining Eq. (24), use has been made of $A_{z}=\beta_{b} \phi$ to express $\nabla\left(\phi-v_{z} A_{z} / c\right)=(1-$ $\left.\beta_{b} v_{z} / c\right) \nabla \phi$. Furthermore, from Eqs. (2) and (21), Poisson's equation can be expressed as

$$
\nabla_{\perp}^{2} \phi+\frac{\partial}{\partial z}\left(\frac{\partial \phi}{\partial z}+\frac{1}{c} \beta_{b} \frac{\partial \phi}{\partial t}\right)=-4 \pi e_{b} \int d^{3} P f_{b}(\mathbf{x}, \mathbf{P}, t)
$$

where $\nabla_{\perp}^{2}=\partial^{2} / \partial x^{2}+\partial^{2} / \partial y^{2}$. The Vlasov-Poisson equations (24) and (25), valid in the laboratory frame, are fully equivalent to the Vlasov-Poisson equations (9) and (15) obtained in the beam frame in the electrostatic approximation. Of course when the two frames coincide $\left(\beta_{b}=0, \gamma_{b}=1, \phi=\phi^{\prime}\right.$ and $\left.A_{z}=A_{z}^{\prime}=0\right)$, Eqs. (24) and (25) are identical in form to Eqs. (9) and (15), as expected.

As noted earlier, because the particle motion is nonrelativistic in the beam frame, it is often advantageous to solve Eqs. (9) and (15) directly, rather than Eqs. (24) and (25). Nonetheless, with some ancillary approximations, the laboratory-frame Vlasov-Poisson equations also simplify further. For example, if the axial velocity spread around $v_{z}=V_{b}=\beta_{b} c$ is very small $\left(\left|\Delta v_{z}\right| \gamma_{b}^{2} \beta_{b} \ll c\right)$, then we approximate $1-v_{z} \beta_{b} c \simeq 1-\beta_{b}^{2}=1 / \gamma_{b}^{2}$, and Eq. (24) reduces to

$$
\frac{\partial f_{b}}{\partial t}+\mathbf{v} \cdot \frac{\partial f_{b}}{\partial \mathbf{x}}+\left[\mathbf{F}_{f o c}-\frac{e_{b}}{\gamma_{b}^{2}} \nabla \phi\right] \cdot \frac{\partial f_{b}}{\partial \mathbf{P}}=0
$$

Equation (26) shows clearly that the magnetic pinching force due to the self-magnetic field in the laboratory frame reduces the electric force by the factor $1 / \gamma_{b}^{2}[1]$. A further simplification occurs in Eq. (25) in circumstances corresponding to an axially continuous beam or very long charge bunch. In Eq. (25), we denote $\partial / \partial x \sim 1 / a$ and $\partial / \partial y \sim 1 / b$ where $a \sim b$ are the transverse beam dimensions; $\partial / \partial z \sim 1 / L$ where $L$ is the characteristic length scale of axial variations; and $\partial / \partial t \sim \omega$ where $\omega^{-1}$ is the characteristic time scale of variations in $\phi(\mathbf{x}, t)$. Then, for $a \sim b$, the terms on the left-hand side of Eq. (25) stand in the ratio

$$
1: \frac{a^{2}}{L^{2}}: \frac{a^{2}}{L^{2}} \frac{\omega L}{c} \beta_{b}
$$

Then for $a^{2} \ll L^{2}$, and even for moderately high frequency with $|\omega L / c| \sim 1$ or $|\omega L / c| \sim \beta_{b}$, the second and third terms on the left-hand side of Eq. (25) are negligibly small, and Poisson's equation can be approximated by 


$$
\nabla_{\perp}^{2} \phi=-4 \pi e_{b} \int d^{3} P f_{b}(\mathbf{x}, \mathbf{P}, t)
$$

where $\nabla_{\perp}^{2}=\partial^{2} / \partial x^{2}+\partial^{2} / \partial y^{2}$. Equations (26) and (28) are similar to the laboratory-frame Vlasov-Poisson equations widely used in the literature [29] to describe thin beam propagation in the paraxial approximation. Approximate Vlasov-Maxwell equations similar to Eqs. (26) and (28) can also be derived using the Darwin-approximation model [31-35] developed by Lee, et al. [30] for intense beam propagation.

\section{CONCLUSIONS}

In summary, in this paper we have developed a clear procedure for solving the nonlinear Vlasov-Maxwell equations for a one-component intense charged particle beam or finite-length charge bunch propagating through a cylindrical conducting pipe (radius $r=r_{w}=$ const.), and confined by an applied focusing force $\mathbf{F}_{\text {foc }}$. In particular, the nonlinear Vlasov-Maxwell equations were Lorentz-transformed to be beam frame ('primed' variables) moving with axial velocity $V_{b}=\beta_{b} c=$ const. relative to the laboratory. In the beam frame, the particle motions are nonrelativistic for the applications of practical interest, already a major simplification. Then, in the beam frame, we made the electrostatic approximation $\left(\mathbf{E}_{s}^{\prime}=-\nabla^{\prime} \phi^{\prime}, \mathbf{E}_{T}^{\prime} \simeq 0 \simeq\right.$ $\mathbf{B}_{s}^{\prime}$ ) which fully incorporates beam space-charge effects, but neglects any fast electromagnetic processes with transverse polarization (e.g., light waves). The resulting Vlasov-Maxwell equations were then Lorentz-transformed back to the laboratory frame, and properties of the self-generated fields and resulting nonlinear Vlasov-Maxwell equations in the laboratory frame were discussed.

\section{ACKNOWLEDGMENTS}

This research was supported by the U.S. Department of Energy. 


\section{REFERENCES}

[1] R. C. Davidson and H. Qin, Physics of Intense Charged Particle Beams in High Energy Accelerators (World Scientific, Singapore, 2001).

[2] S. Y. Lee, Accelerator Physics (World Scientific, Singapore, 1999).

[3] A. W. Chao, Physics of Collective Beam Instabilities in High Energy Accelerators (John Wiley \& Sons, Inc., New York, 1993).

[4] M. Reiser, Theory and Design of Charged Particle Beams (John Wiley \& Sons, Inc., New York, 1994).

[5] T. P. Wangler, Principles of RF Linear Accelerators (John Wiley \& Sons, Inc., New York, 1998).

[6] H. Wiedemann, Particle Accelerator Physics I: Basic Principles and Linear Beam Dynamics (Springer-Verlag, New York, 1999).

[7] H. Wiedemann, Particle Accelerator Physics II: Nonlinear and Higher-Order Beam Dynamics (Springer-Verlag, New York, 1998).

[8] See, for example, Proceedings of the 1999 Particle Accelerator Conference (IEEE Catalog Number 99CH36366), pp. 1-3778.

[9] See, for example, Proceedings of the International Heavy Ion Fusion Symposium, Nuclear Instruments and Methods in Physics Research A464, pp. 1-674 (2001).

[10] I. M. Kapchinskij and V. V. Vladimirskij, in Proceedings of the International Conference on High Energy Accelerators and Instrumentation (CERN Scientific Information Service, Geneva, 1959), p. 274.

[11] R. L. Gluckstern, in Proceedings of the 1970 Proton Linear Accelerator Conference, Batavia, IL, edited by M. R. Tracy (National Accelerator Laboratory, Batavia, IL, 1971), p. 811. 
[12] I. Hofmann, L. J. Laslett, L. Smith and I. Haber, Particle Accelerators 13, 145 (1983).

[13] T. -S. Wang and L. Smith, Particle Accelerators 12, 247 (1982).

[14] J. Struckmeier, J. Klabunde and M. Reiser, Particle Accelerators 15, 47 (1984).

[15] I. Hofmann and J. Struckmeier, Particle Accelerators 21, 69 (1987).

[16] J. Struckmeier and I. Hofmann, Particle Accelerators 39, 219 (1992).

[17] C. Chen and R. C. Davidson, Phys. Rev. E49, 5679 (1994).

[18] R. L. Gluckstern, W. -H. Cheng and H. Ye, Phys. Rev. Lett. 75, 2835 (1995).

[19] N. Brown and M. Reiser, Phys. Plasmas 2, 965 (1995).

[20] R. C. Davidson and C. Chen, Particle Accelerators 59, 175 (1998).

[21] R. C. Davidson and H. Qin, Physical Review Special Topics on Accelerators and Beams 2, 114401 (1999).

[22] R. C. Davidson, W. W. Lee and P. Stoltz, Phys. Plasmas 5, 279 (1998).

[23] R. C. Davidson, Phys. Rev. Lett. 81, 991 (1998).

[24] R. C. Davidson, Phys. Plasmas 5, 3459 (1998).

[25] P. H. Stoltz, R. C. Davidson, and W. W. Lee, Phys. Plasmas 6, 298 (1999).

[26] P. J. Channell, Phys. Plasmas 6, 982 (1999).

[27] H. Qin, R. C. Davidson and W. W. Lee, Physical Review Special Topics on Accelerators and Beams 3, 084401 (2000); 3, 109901 (2000).

[28] R. C. Davidson, H. Qin and P. J. Channell, Physical Review Special Topics on Accelerators and Beams 2, 074401 (1999); 3, 029901 (2000).

[29] See, for example, Chapters 5-8 of Ref. 1. 
[30] W. W. Lee, E. Startsev, H. Qin and R. C. Davidson, Proceedings of the 2001 Particle Accelerator Conference, in press (2001).

[31] C. W. Nielson and H. R. Lewis, Methods in Computational Physics (Academic Press, New York, 1976), Vol. 16, p. 976.

[32] R. C. Davidson, D. A. Hammer, I. Haber, and C. E. Wagner, Phys. Fluids 15, 317 (1972).

[33] Busnardo-Neto, et al., J. Comput. Phys. 23, 300 (1977).

[34] W. W. Lee, M. S. Chance and H. Okuda, Phys. Rev. Lett. 46, 1675 (1981).

[35] C. G. Darwin, Phil. Mag. 39, 537 (1920).

[36] L. D. Landau and E. M. Lifshitz, The Classical Theory of Fields (Pergamon, New York, 1971).

[37] J. D. Jackson, Classical Electrodynamics (John Wiley and Sons, Inc., New York, 1975). 


\section{External Distribution}

Plasma Research Laboratory, Australian National University, Australia

Professor I.R. J ones, Flinders University, Australia

Professor J oão Canalle, Instituto de Fisica DEQ/IF - UERJ , Brazil

Mr. Gerson O. Ludwig, Instituto Nacional de Pesquisas, Brazil

Dr. P.H. Sakanaka, Instituto Fisica, Brazil

The Librarian, Culham Laboratory, England

Library, R61, Rutherford Appleton Laboratory, England

Mrs. S.A. Hutchinson, JET Library, England

Professor M.N. Bussac, Ecole Polytechnique, France

Librarian, Max-Planck-Institut für Plasmaphysik, Germany

J olan Moldvai, Reports Library, MTA KFKI-ATKI, Hungary

Dr. P. Kaw, Institute for Plasma Research, India

Ms. P.J . Pathak, Librarian, Insitute for Plasma Research, India

Ms. Clelia De Palo, Associazione EURATOM-ENEA, I taly

Dr. G. Grosso, Instituto di Fisica del Plasma, Italy

Librarian, Naka Fusion Research Establishment, J AERI, J apan

Library, Plasma Physics Laboratory, Kyoto University, J apan

Research Information Center, National Institute for Fusion Science, J apan

Dr. O. Mitarai, Kyushu Tokai University, J apan

Library, Academia Sinica, Institute of Plasma Physics, People's Republic of China

Shih-Tung Tsai, Institute of Physics, Chinese Academy of Sciences, People's Republic of China

Dr. S. Mirnov, TRINITI, Troitsk, Russian Federation, Russia

Dr. V.S. Strelkov, Kurchatov Institute, Russian Federation, Russia

Professor Peter Lukac, Katedra Fyziky Plazmy MFF UK, Mlynska dolina F-2, Komenskeho Univerzita, SK-842 15 Bratislava, Slovakia

Dr. G.S. Lee, Korea Basic Science Institute, South Korea

Mr. Dennis Bruggink, Fusion Library, University of Wisconsin, USA

Institute for Plasma Research, University of Maryland, USA

Librarian, Fusion Energy Division, Oak Ridge National Laboratory, USA

Librarian, Institute of Fusion Studies, University of Texas, USA

Librarian, Magnetic Fusion Program, Lawrence Livermore National Laboratory, USA

Library, General Atomics, USA

Plasma Physics Group, Fusion Energy Research Program, University of California at San Diego, USA

Plasma Physics Library, Columbia University, USA

Alkesh Punjabi, Center for Fusion Research and Training, Hampton University, USA

Dr. W.M. Stacey, Fusion Research Center, Georgia Institute of Technology, USA

Dr. J ohn Willis, U.S. Department of Energy, Office of Fusion Energy Sciences, USA

Mr. Paul H. Wright, Indianapolis, Indiana, USA 
The Princeton Plasma Physics Laboratory is operated by Princeton University under contract with the U.S. Department of Energy.

\author{
Information Services \\ Princeton Plasma Physics Laboratory \\ P.O. Box 451 \\ Princeton, NJ 08543
}

Phone: 609-243-2750

Fax: 609-243-2751

e-mail: pppl_info@pppl.gov

Internet Address: http://www.pppl.gov 